\title{
Mutated Allele Fraction(MAF) of Epigenetically Driven Target Genes, A Biomarker Assay to Support Clinical Management in Solid Tumors Treated with Multi Targeted Epigenetic Therapy(MTET)
}

\author{
Mohammad Amin Nezami* \\ President and CEO, Sahel Oncology LLC, Pacific Medical Center of Hope, USA
}

*Corresponding author: Mohammad Amin Nezami, President and CEO, Sahel Oncology

Received Date: September 13, 2019

LLC, Pacific Medical Center of Hope, USA.

Keywords: Multi targeted epigenetic therapy; liquid biopsy; circulating DNA

\section{Research Protocol}

\section{Background}

Our current understanding of the epigenetic science has transformed our ability to deliver therapies more precisely to the epigenetic targets driving the tumor growth and disrupting its behavior. In concert with our efforts to regulate the transcription of altered genes involved in tumor biology, we have emphasized a range of epigenetically regulated genes that control the tumor key molecular targets, involved with its growth and metastasis.

\section{Methods}

173 cases treated by epigenetic therapy were collectively selected. 64 percent of all cases were stage four and had no other viable options. This population consisted of 65 percent female and 35 percent male, with age range of 28 to 79.66 patients had one sample and 63 patients had at least two samples. Treatment consisted of natural polyphenols (quercetin formulation) provided as supportive care. Doses were $1500 \mathrm{mg} / \mathrm{m} 2$ BSA IV, weekly. The schedule of therapy was variable depending on the tumor type and staging. Treatment duration depended on tumor stage and state of disease, from two weeks to several months. The most common tumor type was breast cancer (134 cases) and least type was Glioblastoma multiforme,(4 cases). The biomarker assays were performed through liquid biopsy(374 samples). Detection rate was 86 percent. 300 samples were positive for circulating DNA. 74 were negative. Response rate was measured by standard clinical and imaging (RECIST) criteria or other surrogate markers for survival. Quality of life measures were assessed by Eastern Cooperative Oncology Group (ECOG) performance scores.

\section{Result}

Statistical analysis on mutated allele fraction (MAF) of epigenetically driven target genes showed correlation with improved outcome in quality and quantity of life compared to historical control. There were 86 percent improved ECOG score reported with a mean of two points.

\section{Conclusion}

Serial monitoring of mutated allele fraction in circulating DNA analysis is feasible and clinically meaningful. We conclude that such biomarker based epigenetic approach in cancer therapy could enhance the current models of supportive care.

\section{Acknowledgement}

Christian Klowsowski, Steven Jeffrey Hager; Research Cancer Institutes of America, Sahel Oncology, Newport Beach, CA; Guardant health, Genomic Specialist, Medical Affairs, Redwood City, CA; California Cancer Associates for Research and Excellence, Fresno, CA.

\section{Conflict of Interest}

No conflict of interest. 